\title{
Sensibilidade dos espermatozoides de dourado (Salminus brasiliensis) a diferentes soluções crioprotetoras
}

[Sensitivity of dourado (Salminus brasiliensis) spermatozoa to different cryoprotectant solutions]

A.T.M. Viveiros, A.V. Oliveira, A.N. Maria, L.H. Orfão, J.C. Souza

Departamento de Zootecnia - UFLA

Caixa Postal 37

37200-000 - Lavras, MG

\begin{abstract}
RESUMO
Em três experimentos, avaliou-se a sensibilidade dos espermatozoides de dourado (Salminus brasiliensis) a diferentes soluções crioprotetoras. No experimento 1, o sêmen foi diluído, 1:10, em 12 soluções (quatro diluidores $\mathrm{x}$ três crioprotetores - dimetilsulfóxido (DMSO), metilglicol ou glicerol). Metade de cada amostra foi resfriada por uma hora e a outra, criopreservada. A motilidade espermática foi avaliada imediatamente após a diluição e após o resfriamento em todas as amostras e, após o descongelamento, apenas nas amostras criopreservadas em DMSO. No experimento 2, o sêmen foi diluído, 1:5, em cinco soluções contendo DMSO e resfriado, criopreservado e avaliado como no experimento 1. No experimento 3, o sêmen foi diluído, 1:5, em quatro soluções contendo DMSO e criopreservado e avaliado quanto à motilidade e à fertilidade. Quando o sêmen foi diluído 1:10, observou-se motilidade acima de 58\% em todas as amostras resfriadas em DMSO e em $\mathrm{NaCl}$-tris-metilglicol. Baixa motilidade foi observada nas amostras resfriadas nas outras combinações com metilglicol (5-32\%) ou glicerol (0-8\%) e naquelas criopreservadas (16-20\%). Todas as amostras diluídas 1:5 apresentaram motilidade de $65-72 \%$ após o resfriamento e de $45-66 \%$ após o descongelamento (experimentos 2 e 3). As taxas de eclosão produzidas com sêmen criopreservado, entretanto, foram baixas (17-23\%) em relação ao sêmen fresco $(60 \%)$.
\end{abstract}

Palavras-chave: dourado, Salminus brasiliensis, diluidor, crioprotetor, criopreservação, fertilização

\begin{abstract}
The sensitivity of dourado (Salminus brasiliensis) spermatozoa to different cryoprotectant solutions was evaluated in three experiments. In experiment 1, semen was diluted, 1:10, in 12 solutions (four extenders $x$ three cryoprotectants - dimethylsulphoxide (DMSO), methyglycol, or glycerol). Half of each sample was refrigerated for one hour while the other half was cryopreserved. Sperm motility was immediately assessed after dilution and after refrigeration in all samples, and after thawing in those cryopreserved in DMSO. In experiment 2, semen was diluted, 1:5, in five solutions containing DMSO, refrigerated, cryopreserved, and analyzed as in experiment 1. In experiment 3, semen was diluted, 1:5, in five solutions containing DMSO, cryopreserved and evaluated for motility and fertility. When semen was diluted 1:10, motility higher than 58\% was observed in all samples refrigerated in DMSO and in NaCl-tris-methylglycol. Low motility was observed in samples refrigerated in the other combinations of methylglycol (5-32\%) or glycerol (0-8\%) and in those cryopreserved (16-20\%). All samples diluted 1:5 yielded motility of 65-72\% after refrigeration, and $45-66 \%$ after thawing (experiments 2 and 3). The hatching rates produced with cryopreserved semen, however, were lower (17-23\%) compared to fresh semen (60\%).
\end{abstract}

Keywords: dourado, Salminus brasiliensis, extender, cryoprotectant, cryopreservation, fertilization

\section{INTRODUÇÃO}

O dourado, Salminus brasiliensis, é um peixe de grande porte, pertencente à bacia do rio Grande e pode atingir mais de um metro de comprimento. A reprodução ocorre de novembro a fevereiro, quando indivíduos dessa espécie realizam migrações reprodutivas em um fenômeno

Recebido em 2 de julho de 2008

Aceito em 8 de abril de 2009

E-mail: ana.viveiros@ufla.br 
conhecido como piracema (Guia..., 2000). O dourado tem alto valor econômico e, por isso, é bastante visado pelos pescadores. Devido às modificações ambientais provocadas pelo homem, como a poluição da água, o desmatamento e a construção de barragens, além da pesca e da captura em época de desova, a população de dourados, assim como a de várias outras espécies de peixes no Brasil, vem diminuindo gradativamente (Carolsfeld e Harvey, 1999).

Com base nesses fatos, torna-se imperiosa e urgente a adoção de práticas de conservação genética. A constituição de bancos de germoplasma de espécies ameaçadas tem sido uma medida eficiente adotada em vários países, pela sua relativa simplicidade e pelo baixo custo de implantação e de manutenção. Sua eventual utilização na restauração da variabilidade genética de estoques debilitados e sob ameaça de extinção iminente faz desta tecnologia uma poderosa ferramenta de conservação de recursos genéticos de peixes (Viveiros e Godinho, 2009).

A criopreservação do sêmen é uma técnica que exige solução crioprotetora adequada e taxas de congelamento e de descongelamento ótimas (Viveiros, 2005). As soluções crioprotetoras são compostas por um diluidor e um crioprotetor intracelular, com a função de prevenir as crioinjúrias dos espermatozoides durante os procedimentos de congelamento e de descongelamento, além de manter os espermatozoides imóveis durante o armazenamento. Os diluidores podem ser soluções simples à base de $\mathrm{NaCl}$ ou de glicose, ou soluções mais complexas numa combinação de vários sais e açúcares, como o diluidor Beltsville Thawing solution $\left(\right.$ BTS $^{\circledR}$, Minitub ${ }^{\circledR}$ ) e a água de coco (Viveiros e Godinho, 2009). O crioprotetor intracelular mais testado e que tem proporcionado bons resultados em espécies nativas é o dimetilsulfóxido (DMSO). Mais recentemente, o metilglicol foi testado em algumas espécies como crioprotetor e produziu resultados semelhantes e, às vezes, até superiores ao DMSO em sêmen de piracanjuba Brycon orbignyanus (Maria et al., 2006b), pirapitinga Brycon nattereri (Oliveira et al., 2007) e curimba Prochilodus lineatus (Viveiros et al., 2009).

Diversas metodologias de criopreservação de sêmen de peixe têm sido testadas em várias espécies, embora o grau de sucesso seja muito variável. A qualidade espermática tem sido avaliada frequentemente, apenas em termos de motilidade após o descongelamento. No entanto, a capacidade de fertilização é o parâmetro mais importante a ser avaliado, embora ovócitos de boa qualidade nem sempre estejam disponíveis. As taxas de fertilização obtidas com sêmen criopreservado estão, cada vez mais, se aproximando das observadas com sêmen fresco (Ribeiro e Godinho, 2003; Viveiros e Maria, 2008; Viveiros et al., 2009).

O presente trabalho foi realizado com o objetivo de avaliar a sensibilidade de espermatozoides de dourado a diferentes soluções crioprotetoras compostas por diluidores simples ou mais complexos, combinados aos crioprotetores intracelulares DMSO, metilglicol e glicerol.

\section{MATERIAL E MÉTODOS}

O trabalho foi desenvolvido durante a piracema, nos meses de janeiro e fevereiro de 2005 e de 2006. Utilizou-se o sêmen de 11 machos de dourado, Salminus brasiliensis, oriundos da Estação de Hidrobiologia e Piscicultura de Furnas Centrais Elétricas, município de São José da Barra, MG, e uma fêmea e um macho provenientes da Estação de Piscicultura da Companhia Energética de Minas Gerais, município de Itutinga, MG. Dos reprodutores capturados com rede de arrasto, em tanque de terra, foram selecionados aqueles que liberavam sêmen sob leve massagem da cavidade celomática no sentido craniocaudal. Depois de pesados, os machos receberam dose única de extrato bruto de hipófise de carpa $(1 \mathrm{mg} / \mathrm{kg}$ de peso corporal), para facilitar a coleta do sêmen. $\mathrm{O}$ sêmen foi coletado em tubos de ensaio graduados e estéreis, e mantido a $4-6^{\circ} \mathrm{C}$, durante as análises. As amostras de sêmen de cada macho foram observadas sob microscópio de luz, descartando-se as que apresentavam motilidade espontânea (autoativação) por contaminação com água ou urina. A motilidade do sêmen foi avaliada após ativação com $\mathrm{NaCl} \quad 50 \mathrm{mM}$ (1 sêmen:5 ativador; Maria et al., 2006a) e estimada como porcentagem de espermatozoides móveis em relação ao total observado. A concentração espermática foi estimada por meio de uma câmara hematimétrica Neubauer Improved. 
O botijão de vapor de nitrogênio, conhecido como dry shipper ${ }^{1}$ foi utilizado como congelador de sêmen.

No experimento 1 , foi avaliado o efeito de diferentes soluções crioprotetoras na motilidade espermática do sêmen, após o resfriamento e a criopreservação. Foram preparadas 12 soluções compostas pela combinação de quatro diluidores e três crioprotetores. Os diluidores testados foram: $\mathrm{NaCl} 154 \mathrm{mM}, \mathrm{NaCl} 200 \mathrm{mM}, \mathrm{NaCl}$ $200 \mathrm{mM}$-tris e BTS ${ }^{\circledR 2}$ glicose $4 \%$, citrato de sódio $0,63 \%$, EDTA $0,13 \%$, sulfato de gentamicina $\left.0,025 \%, \mathrm{NaHCO}_{3} \quad 0,13 \%, \mathrm{KCl} \quad 0,08 \%\right)$. Os crioprotetores testados foram: DMSO, metilglicol e glicerol. O sêmen de cada macho $(\mathrm{n}=3)$ foi diluído, 1:10 (sêmen:volume final), em cada uma das 12 soluções, na proporção final de 1 sêmen:8 diluidor:1 crioprotetor. Após a diluição, metade do volume de cada amostra foi resfriada $\left(4-6^{\circ} \mathrm{C}\right)$ em tubos de ensaio de $5 \mathrm{~mL}$, por 1 hora e a outra metade envasada em palhetas de $0,5 \mathrm{~mL} \quad(\mathrm{n}=3$ palhetas/solução/ macho), vedadas com esfera plástica, congeladas em vapor de nitrogênio, a $-170^{\circ} \mathrm{C}$, por 24 horas e armazenadas em nitrogênio líquido. Uma amostra de sêmen de cada macho foi resfriada sem diluição e considerada como controle. Em todas as amostras, a motilidade espermática foi avaliada imediatamente após a diluição e após uma hora de resfriamento. Apenas as amostras congeladas em DMSO foram descongeladas em banho-maria, a $60^{\circ} \mathrm{C}$, por oito segundos, e avaliadas quanto à motilidade, da mesma forma descrita para o sêmen fresco.

No experimento 2, o sêmen de cada macho $(\mathrm{n}=3)$ foi diluído, 1:5 (sêmen:volume final), na proporção 1 sêmen:3 diluidor:1 crioprotetor, em solução contendo DMSO e um dos seguintes diluidores: $\mathrm{NaCl} 154 \mathrm{mM}, \mathrm{NaCl} 200 \mathrm{mM}, \mathrm{NaCl}$ $200 \mathrm{mM}$-tris, glicose $277 \mathrm{mM}$ ou BTS ${ }^{\circledR}$. Após a diluição, metade das amostras de sêmen foi imediatamente resfriada e a outra, envasada em palhetas $(n=3$ palhetas/solução/macho $) \quad e$ criopreservada, como no experimento 1. Uma amostra de sêmen de cada macho foi resfriada sem diluição e considerada como controle. A motilidade espermática foi avaliada em todas as amostras imediatamente após a diluição, após o

\footnotetext{
${ }^{1}$ Taylor Wharton, modelo CP 100 - Theodore, EUA.

${ }^{2}$ Minitub - Tiefenbach, Alemanha.
}

resfriamento e após o descongelamento do sêmen.

No experimento 3, o sêmen criopreservado em diferentes soluções foi avaliado quanto às taxas de motilidade e de eclosão. O sêmen de cada macho $(\mathrm{n}=5)$ foi diluído, 1:5, em solução contendo DMSO combinado a um dos seguintes diluidores: $\mathrm{NaCl} 154 \mathrm{mM}, \mathrm{NaCl} 200 \mathrm{mM}$, glicose $277 \mathrm{mM}$ ou $\mathrm{BTS}^{\circledR}$. O diluidor $\mathrm{NaCl} 200 \mathrm{mM}$-tris, por apresentar composição química e resultados semelhantes ao $\mathrm{NaCl} 200 \mathrm{mM}$, não foi reavaliado. Após diluição, o sêmen foi criopreservado $(\mathrm{n}=6$ palhetas/solução/macho) de acordo com a metodologia descrita anteriormente. Em todas as amostras, a motilidade espermática pósdescongelamento foi avaliada em três palhetas de cada solução. Apenas as amostras congeladas em glicose e em BTS ${ }^{\circledR}$ foram usadas para fertilizar ovócitos. Para isso, uma fêmea recebeu duas doses de extrato bruto de hipófise de carpa ( 0,5 e $5 \mathrm{mg} / \mathrm{kg}$ de peso corporal), com intervalo de 12 horas, para indução da desova, seguindo o protocolo usado na estação. $\mathrm{O}$ sêmen descongelado foi imediatamente rediluído em $\mathrm{NaCl} 200 \mathrm{mM}$, na proporção final 1:10. Uma alíquota de $100 \mu \mathrm{L}$ de sêmen rediluído de cada palheta de cada macho foi misturada em $0,15 \mathrm{~g}$ de ovócitos (aproximadamente 200 ovócitos), e a fertilização foi iniciada após adição de $2 \mathrm{~mL}$ de água dos aquários em que os reprodutores se encontravam. Decorridos dois minutos de leve agitação, foram acrescentados mais $10 \mathrm{~mL}$ de água para a hidratação dos ovócitos e deixados em repouso por mais cinco minutos. Os ovos foram, então, transferidos para incubadoras de PVC teladas no fundo (Viveiros et al., 2000) e mantidos em água com temperatura média de $26^{\circ} \mathrm{C}$. Como controle, foi utilizado sêmen fresco de outro macho, diluído em $\mathrm{NaCl} 200 \mathrm{mM}$, na proporção 1:200, no início e no final desse experimento, para controlar a qualidade dos ovócitos. A taxa de eclosão foi verificada 20 horas após a fertilização e expressa por: $\left(\mathrm{n}^{\circ}\right.$ de larvas $\mathrm{x} 100) /\left(\mathrm{n}^{\circ}\right.$ de larvas + ovos).

Os dados observados estão apresentados como média \pm erro-padrão e, por serem expressos em porcentagens, tiveram os resíduos de cada modelo testados quanto à distribuição normal. Os valores que não apresentaram distribuição normal foram transformados em arc seno $\sqrt{ } \mathrm{X}$ e, então, submetidos à análise de variância. As médias foram comparadas pelo teste de Scott- 
Knott, a 5\% de probabilidade, utilizando-se o pacote computacional SISVAR (Ferreira, 1999).

\section{RESULTADOS}

O sêmen obtido dos 11 machos de dourado variou de branco-leitoso a ligeiramente amarelado, com pouca viscosidade. $\mathrm{O}$ volume seminal médio coletado foi $13,1 \pm 3,2 \mathrm{~mL}$ e a concentração espermática foi $6,2 \times 10^{9} \pm 1,6 \times 10^{9}$ espermatozoides $/ \mathrm{mL}$ de sêmen.

O botijão de vapor de nitrogênio usado como congelador das amostras de sêmen estabilizou-se à temperatura de $-172^{\circ} \mathrm{C}$, após o período de 270 segundos, resultando na velocidade média de congelamento de $-44,1^{\circ} \mathrm{C} /$ minutos, entre $+26^{\circ} \mathrm{C}$ e $-172^{\circ} \mathrm{C}$.

No experimento 1, as amostras de sêmen diluídas nas soluções contendo DMSO como crioprotetor e resfriadas por uma hora apresentaram as maiores taxas de motilidade espermática, semelhante ao sêmen não diluído (controle) (Tab. 1). Quando o sêmen foi diluído nas soluções que continham metilglicol ou glicerol, houve redução na motilidade espermática, com exceção do sêmen diluído em $\mathrm{NaCl}$ 200mM-tris combinado ao metilglicol (73\%). O sêmen criopreservado apresentou baixa motilidade espermática após o descongelamento (16\%$20 \%$ ).

No experimento 2, não foram observadas diferenças significativas $(\mathrm{P}>0,05)$ na motilidade espermática do sêmen após o resfriamento $(65 \%$ $77 \%$ ) ou após a criopreservação (45\%-66\%), nas diferentes soluções contendo DMSO (Tab. 2).

No experimento 3, as maiores taxas de motilidade espermática $(>60 \%)$ foram observadas nas amostras de sêmen criopreservadas em glicose e em BTS $^{\circledR}$, em relação às amostras congeladas nas soluções da $\mathrm{NaCl}$ (45\%-49\%; Tab. 3). A capacidade de fertilização dos espermatozoides após o descongelamento foi reduzida (17\%-23\%) em relação ao sêmen fresco (60\%). A partir do valor médio da concentração espermática estimada no sêmen dos dourados utilizados nesse experimento, a relação espermatozoide:ovócito utilizada na fertilização foi calculada em $28,7 \times 10^{5}$ espermatozoides criopreservados por ovócito e $1,4 \times 10^{5}$ espermatozoides frescos por ovócito.

Tabela 1. Motilidade espermática (média \pm erro-padrão) do sêmen de dourado diluído 1:10 (sêmen:volume final), em diferentes soluções e avaliado imediatamente após a diluição (fresco), após uma hora de resfriamento e após o descongelamento

\begin{tabular}{|c|c|c|c|c|}
\hline \multirow[t]{2}{*}{$\begin{array}{l}\text { Crioprotetor } \\
(10 \%)\end{array}$} & \multirow{2}{*}{ Diluidor } & \multicolumn{3}{|c|}{ Motilidade espermática (\%) } \\
\hline & & Fresco $^{2}$ & Resfriado $^{2}$ & Criopreservado $^{3}$ \\
\hline \multirow[t]{4}{*}{ DMSO } & $\mathrm{NaCl} 154 \mathrm{mM}$ & $80 \pm 8 \mathrm{a}$ & $58 \pm 8 \mathrm{a}$ & $16 \pm 9 a$ \\
\hline & $\mathrm{NaCl} 200 \mathrm{mM}$ & $80 \pm 5 \mathrm{a}$ & $73 \pm 8 \mathrm{a}$ & $20 \pm 5 \mathrm{a}$ \\
\hline & $\mathrm{NaCl} 200 \mathrm{mM}$-tris & $60 \pm 16 a$ & $78 \pm 3 a$ & $16 \pm 9 \mathrm{a}$ \\
\hline & $\mathrm{BTS}^{\circledR 1}$ & $90 \pm 0 \mathrm{a}$ & $78 \pm 5 \mathrm{a}$ & $16 \pm 9 \mathrm{a}$ \\
\hline \multirow[t]{4}{*}{ Metilglicol } & $\mathrm{NaCl} 154 \mathrm{mM}$ & $38 \pm 16 b$ & $5 \pm 2 b$ & --- \\
\hline & $\mathrm{NaCl} 200 \mathrm{mM}$ & $62 \pm 19 \mathrm{a}$ & $32 \pm 23 b$ & --- \\
\hline & $\mathrm{NaCl} 200 \mathrm{mM}$-tris & $80 \pm 5 \mathrm{a}$ & $73 \pm 5 \mathrm{a}$ & --- \\
\hline & $\mathrm{BTS}^{\circledR 1}$ & $60 \pm 13 a$ & $21 \pm 8 b$ & --- \\
\hline \multirow[t]{4}{*}{ Glicerol } & $\mathrm{NaCl} 154 \mathrm{mM}$ & $20 \pm 3 b$ & $0 \pm 1 b$ & --- \\
\hline & $\mathrm{NaCl} 200 \mathrm{mM}$ & $38 \pm 19 b$ & $8 \pm 8 b$ & --- \\
\hline & $\mathrm{NaCl} 200 \mathrm{mM}$-tris & $25 \pm 8 b$ & $7 \pm 1 b$ & --- \\
\hline & $\mathrm{BTS}^{\circledR 1}$ & $48 \pm 20 b$ & $0 \pm 1 b$ & --- \\
\hline \multicolumn{2}{|c|}{ Sêmen fresco não diluído } & $85 \pm 3 a$ & $87 \pm 3 \mathrm{a}$ & --- \\
\hline
\end{tabular}


Tabela 2. Motilidade espermática (média \pm erro-padrão) do sêmen de dourado após diluição (fresco), resfriamento (1 hora) e criopreservação, em diferentes soluções crioprotetoras contendo DMSO (diluição $1: 5)$

\begin{tabular}{lccc}
\hline \multirow{2}{*}{ Diluidor } & \multicolumn{3}{c}{ Motilidade espermática (\%)* $^{*}$} \\
\cline { 2 - 4 } $\mathrm{NaCl} 154 \mathrm{mM}$ & Fresco $^{2}$ & Resfriado $^{2}$ & Criopreservado $^{3}$ \\
$\mathrm{NaCl} 200 \mathrm{mM}$ & $68 \pm 9$ & $65 \pm 10$ & $66 \pm 9$ \\
$\mathrm{NaCl} 200 \mathrm{mM}$-tris & $72 \pm 5$ & $72 \pm 6$ & $53 \pm 11$ \\
Glicose 277mM & $70 \pm 6$ & $72 \pm 3$ & $45 \pm 11$ \\
BTS $^{\circledR}$ 1 & $78 \pm 5$ & $77 \pm 3$ & $47 \pm 16$ \\
Sêmen fresco não diluído & $73 \pm 3$ & $70 \pm 5$ & $50 \pm 17$ \\
\hline
\end{tabular}

*Médias não diferiram entre si pelo teste $\mathrm{F}(\mathrm{P}>0,05)$.

${ }^{1}$ Glicose $4 \%$, citrato de sódio $0,635 \%$, EDTA $0,13 \%$, sulfato de gentamicina $0,025 \%$, $\mathrm{NaHCO}_{3} 0,13 \%, \mathrm{KCl} 0,08 \%$.

${ }^{2} \mathrm{n}=3$ machos.

${ }^{3} \mathrm{n}=3$ machos $\mathrm{x} 3$ palhetas.

Tabela 3. Motilidade espermática e taxa de eclosão (média \pm erro-padrão) do sêmen de dourado criopreservado em diferentes soluções contendo DMSO (diluição 1:5)

\begin{tabular}{lcc}
\hline Diluidores & Motilidade espermática $^{3}(\%)$ & Eclosão $^{3}(\%)$ \\
\hline $\mathrm{NaCl} 154 \mathrm{mM}$ & $45 \pm 8 \mathrm{c}$ & -- \\
$\mathrm{NaCl} 200 \mathrm{mM}$ & $49 \pm 3 \mathrm{c}$ & --- \\
Glicose $277 \mathrm{mM}$ & $61 \pm 3 \mathrm{~b}$ & $23 \pm 4 \mathrm{~b}$ \\
$\mathrm{BTS}^{\circledR} 1$ & $64 \pm 2 \mathrm{~b}$ & $17 \pm 4 \mathrm{~b}$ \\
Sêmen fresco diluído $^{2}$ & $99 \pm 1 \mathrm{a}$ & $60 \pm 6 \mathrm{a}$ \\
\hline
\end{tabular}

Médias seguidas por letras diferentes, na mesma coluna, diferem entre si, pelo teste Scott-Knott $(\mathrm{P}<0,05)$.

${ }^{1}$ Glicose $4 \%$, citrato de sódio $0,63 \%$, EDTA $0,13 \%$, sulfato de gentamicina $0,02 \%, \mathrm{NaHCO}_{3} 0,13 \%, \mathrm{KCl} 0,08 \%$

${ }^{2} \mathrm{n}=1$ macho $\times 2$ repetições no início x 2 repetições no final da fertilização.

${ }^{3} \mathrm{n}=5$ machos $\mathrm{x} 3$ palhetas.

\section{DISCUSSÃO}

A velocidade de congelamento ótima, para células espermáticas, varia entre -1 e $-10^{\circ} \mathrm{C} / \mathrm{min}$, para sêmen humano (Henry et al., 1993) a $100^{\circ} \mathrm{C} / \mathrm{min}$, para sêmen de touro (Woelders, 1997). O botijão de vapor de nitrogênio utilizado neste trabalho apresentou velocidade de congelamento de $-44,1^{\circ} \mathrm{C} / \mathrm{min}$, entre $+26^{\circ}$ e $172^{\circ} \mathrm{C}$, estando dentro dos limites de $-10^{\circ}$ a $50^{\circ} \mathrm{C} / \mathrm{min}$, recomendados por Harvey e Carolsfeld (1993). No mesmo tipo de botijão, já foi registrada taxa de congelamento de $45^{\circ} \mathrm{C} / \mathrm{min}$ entre $+8^{\circ}$ e $-150^{\circ} \mathrm{C}$ (Carolsfeld et al., 2003). O uso do botijão de vapor de nitrogênio como congelador de sêmen de dourado foi satisfatório.

A qualidade do sêmen após os processos de congelamento e de descongelamento depende do tipo e da concentração do crioprotetor intracelular, da sua combinação com diferentes diluidores e da taxa de diluição utilizada. Neste estudo, a sensibilidade dos espermatozoides submetidos a diferentes soluções crioprotetoras foi avaliada com base na motilidade espermática, após uma hora de exposição, pois esse período foi considerado o tempo necessário para a manipulação do sêmen para a criopreservação. $\mathrm{O}$ sêmen diluído em soluções crioprotetoras contendo DMSO, em geral, apresentou as maiores motilidades espermáticas após uma hora de exposição e essas foram semelhantes às motilidades observadas no sêmen não diluído. $\mathrm{O}$ DMSO é o crioprotetor mais utilizado na criopreservação de sêmen de várias espécies de peixes tropicais, inclusive em dourado (Cóser et al., 1984; Carolsfeld et al., 2003). O uso do metilglicol como crioprotetor foi testado anteriormente e proporcionou resultados iguais ou superiores ao DMSO, na criopreservação de sêmen de piracanjuba, B. orbignyanus (Maria et al., 2006a,b), de pirapitinga, B. nattereri (Oliveira et al., 2007) e de curimba, $P$. lineatus (Viveiros et al., 2009). No dourado, entretanto, o uso de metilglicol foi eficiente apenas quando as amostras foram diluídas em NaCl-tris. O glicerol 
foi o único crioprotetor testado que, em todas as combinações com os diluidores, reduziu a motilidade espermática imediatamente após a diluição do sêmen. Apesar desse efeito negativo sobre a motilidade espermática do sêmen de dourado, o glicerol foi usado com sucesso na criopreservação de sêmen de bagre africano Clarias gariepinus (Steyn, 1993) e de bagre europeu Silurus glanis (Linhart et al., 1993).

No presente trabalho foram testados diluidores à base de $\mathrm{NaCl}$ e de glicose. Foi possível identificar que as amostras congeladas em soluções contendo glicose (glicose $277 \mathrm{mM}$ e BTS $^{\circledR}$ ) apresentaram maior motilidade do que aquelas congeladas em diluidores salinos. Soluções simples, constituídas apenas de glicose $5 \%$ e DMSO, têm sido usadas com sucesso na criopreservação do sêmen de várias espécies nativas, inclusive do dourado (Carolsfeld et al., 2003). A solução comercial BTS $^{\circledR}$ foi desenvolvida como diluidor de sêmen de suíno. Essa solução possui $80 \%$ de glicose na sua composição e tem sido utilizada como diluidor na criopreservação em várias espécies de peixes nativos, como a piracanjuba, $B$. orbignyanus (Maria et al., 2006a) e a curimba, $P$. lineatus (Viveiros et al., 2009), dentre outras. Esse é o primeiro relato do uso do BTS $^{\circledR}$ como diluidor de sêmen de dourado.

A taxa de diluição sêmen:solução crioprotetora afeta a motilidade espermática após a criopreservação. As diluições utilizadas variam entre autores e entre espécies de peixe. No presente estudo, as maiores motilidades foram observadas nas amostras diluídas na proporção de 1:5, em relação às amostras diluídas 1:10 (sêmen:volume final). Em estudos anteriores com a mesma espécie, a taxa de diluição 1:5 (sêmen:volume final) foi utilizada com sucesso na criopreservação do sêmen (Cóser et al., 1984; Carolsfeld et al., 2003).

Durante a fertilização de ovócitos de peixes, é comum a utilização de um excesso de espermatozoides por ovócito como garantia de fertilização máxima. Quando o sêmen é criopreservado, o número deve ser ainda maior, já que os processos de congelamento e descongelamento provocam grande mortalidade dos espermatozoides, além de danificarem suas estruturas celulares, dificultando a fertilização (Rodriguez-Martinez e Ekwall, 1998). Neste estudo, a proporção estimada foi de $28,7 \times 10^{5}$ espermatozoides congelados por ovócito e de $1,4 \times 10^{5}$ espermatozoides frescos por ovócito. Como a taxa de eclosão obtida com sêmen fresco $(60 \%)$ foi maior que a obtida com sêmen criopreservado (17\%-23\%), é provável que os espermatozoides tenham sofrido danos estruturais que, embora não tenham reduzido acentuadamente a motilidade (61\%-64\%), dificultaram/impediram o processo de fertilização. O sêmen criopreservado de outras espécies de peixes tropicais tem produzido taxas de eclosão dos ovos melhores do que a observada em dourado (esse estudo) e tem se aproximado cada vez mais daquelas obtidas com sêmen fresco. Em matrinxã, Brycon cephalus, o sêmen criopreservado proporcionou $68 \%$ de eclodibilidade em relação ao sêmen fresco (Ninhaus-Silveira et al., 2006). Em piracanjuba, $B$. orbignyanus, observou-se $58 \%-66 \%$ de eclodibilidade com o sêmen criopreservado e $86 \%$ com o sêmen fresco (Maria et al., 2006a).

Os espermatozoides de dourado resistem melhor ao processo de criopreservação quando diluídos em solução contendo DMSO e um diluidor à base de glicose. Os espermatozoides foram altamente sensíveis à exposição ao glicerol, e este crioprotetor não deverá ser usado em soluções crioprotetoras para o sêmen dessa espécie.

\section{AGRADECIMENTOS}

À FAPEMIG, pelo financiamento do projeto (EDT 2224/2003); ao CNPq, pela bolsa de produtividade em pesquisa (302689/2004-4); à Capes, pela bolsa de mestrado (A.V. Oliveira); à Furnas Centrais Hidrelétricas e à CEMIG, unidade Itutinga, por disponibilizarem os reprodutores; ao A. Marchetti, da Minitub ${ }^{\circledR}$, pela doação do BTS $^{\circledR}$ e das palhetas; ao Prof. R.T.F. Freitas, pelo apoio ao desenvolvimento estatístico e aos colegas J.F.A. Koch, Z.A. Izaú e R.V. Araújo, pelo auxílio nas coletas e no processamento do sêmen.

\section{REFERÊNCIAS BIBLIOGRÁFICAS}

CAROLSFELD, J.; GODINHO, H.P.; ZANIBONI FILHO, E. et al. Cryopreservation of sperm in Brazilian migratory fish conservation. J. Fish Biol., v.63, p.472-489, 2003. 
CAROLSFELD, J.; HARVEY, B. Conservação de recursos genéticos de peixes: teoria e prática. In: CURSO de treinamento brasileiro. Victoria, Canadá: World Fisheries Trust, 1999. 47p.

CÓSER, A.M.; GODINHO, H.; RIBEIRO, D.M. Cryogenic preservation of spermatozoa from Prochilodus scrofa and Salminus maxillosus. Aquaculture, v.37, p.387-390, 1984.

FERREIRA, D.F. Sistema de análise de variância (SISVAR). versão 4.3 Lavras: UFLA, 1999. (Build 43).

GUIA ilustrado de peixes da bacia do rio Grande. Belo Horizonte: CEMIG/CETEC, 2000. $141 \mathrm{p}$.

HARVEY, B.; CAROLSFELD, J. Induced breeding in tropical fish culture. Ottawa: International Development Research Centre, 1993. $144 p$.

HENRY, M.A.; NOILES, E.E.; GAO, D.Y. et al. Cryopreservation of human spermatozoa. The effects of cooling rate and warming rate on the maintenance of motility, plasma membrane integrity, and mitochondrial function. Fertil. Steril., v.60, p.911-918, 1993.

LINHART, O.; BILLARD, R.; PROTEAU, J.P. Cryopreservation of European catfish (Silurus glanis L.) spermatozoa. Aquaculture, v.115, p.347-359, 1993.

MARIA, A.N.; VIVEIROS, A.T.M.; FREITAS, R.T.F. et al. Extenders and cryoprotectants for cooling and freezing of piracanjuba (Brycon orbignyanus) semen, an endangered Brazilian teleost fish. Aquaculture, v.260, p.298-306, $2006 a$.

MARIA, A.N.; VIVEIROS, A.T.M.; ORFAO, L.H. et al. Effects of cooling and freezing on sperm motility of the endangered fish piracanjuba Brycon orbignyanus (Characiformes, Characidae). Anim. Reprod., v.3, p.55-60, 2006b.

NINHAUS-SILVEIRA, A.; FORESTI, F.; VERÍSSIMO-SILVEIRA, R. et al. Seminal analysis, cryogenic preservation, and fertility in matrinxã fish, Brycon cephalus (Günther, 1869). Braz. Arch. Biol. Technol., v.49, p.651-659, 2006.
OLIVEIRA, A.V.; VIVEIROS, A.T.M.; MARIA, A.N. et al. Sucesso do resfriamento e congelamento de sêmen em pirapitinga (Brycon nattereri). Arq. Bras. Med. Vet. Zootec., v.59, p.1509-1515, 2007.

RIBEIRO, R.I.M.A.; GODINHO, H.P. Criopreservação do sêmen testicular do teleósteo piau-açu Leporinus macrocephalus. Arq. Bras. Med. Vet. Zootec., v.55, p.75-79, 2003.

RODRIGUEZ-MARTINEZ, H.; EKWALL, H. Electron microscopy in the assessment of cryopreserved spermatozoa viability. Microsc. Anal., v.65, p.11-13, 1998.

STEYN, G.J. The effect of freezing rate on the survival of cryopreserved African sharptooth catfish (Clarias gariepinus) spermatozoa. Cryobiology, v.30, p.581-590, 1993.

VIVEIROS, A.T.M. Semen cryopreservation in catfish species, with particular emphasis on the African catfish. Anim. Breed. Abstr., v.73, p.1N9N, 2005.

VIVEIROS, A.T.M.; GODINHO, H.P. Sperm quality and cryopreservation of Brazilian freshwater fish species: a review. Fish Phys. Bioch., v.35, p.137-150, 2009.

VIVEIROS, A.T.M.; MARIA, A.N. Sêmen cryopreservation of piracanjuba (Brycon orbignyanus), an endangered Brazilian species.. In: CABRITA, E.; ROBLES, V.; HERRAEZ, P. (Ed.). Methods in Reproductive Aquaculture: Marine and Freshwater Species. Boca Raton: CRC Press, 2008. p.361-365.

VIVEIROS, A.T.M.; SO, N.; KOMEN, J. Sperm cryopreservation of African catfish (Clarias gariepinus): cryoprotectants, freezing rates and sperm: egg dilution ratio. Theriogenology, v.54, p.1305-1308, 2000.

VIVEIROS, A.T.M.; ORFÃO, L.H.; MARIA, A.N. et al. A simple, inexpensive and successful freezing method for curimba Prochilodus lineatus (Characiformes) semen. Anim. Reprod. Sci., v.112, p.293-300, 2009.

WOELDERS, H. Fundamentals and recent development in cryopreservation of bull and boar semen. Vet. Q., v.19, p.135-138, 1997. 